\title{
Sur la connaissance et l'usage de la langue dans le Setomaa d'après ELDIA
}

About Language Knowledge and Use in Setomaa According to ELDIA.

Keele oskuse ja kasutuse hinnangutest Setomaal ELDIA peeglis

\section{Kadri Koreinik}

Traducteur : Eva Toulouze

\section{(2) OpenEdition \\ Journals}

Édition électronique

URL : https://journals.openedition.org/efo/13817

DOI : 10.4000/efo. 13817

ISSN : 2275-1947

Éditeur

INALCO

\section{Référence électronique}

Kadri Koreinik, "Sur la connaissance et l'usage de la langue dans le Setomaa d'après ELDIA », Études finno-ougriennes [En ligne], 49-50 | 2018, mis en ligne le 28 janvier 2019, consulté le 08 juillet 2021.

URL : http://journals.openedition.org/efo/13817 ; DOI : https://doi.org/10.4000/efo.13817

Ce document a été généré automatiquement le 8 juillet 2021.

\section{(c) (1) \&}

Études finno-ougriennes est mis à disposition selon les termes de la Licence Creative Commons Attribution - Pas d'Utilisation Commerciale 4.0 International. 


\section{Sur la connaissance et l'usage de la langue dans le Setomaa d'après ELDIA}

About Language Knowledge and Use in Setomaa According to ELDIA. Keele oskuse ja kasutuse hinnangutest Setomaal ELDIA peeglis

\section{Kadri Koreinik}

Traduction : Eva Toulouze

1 Le projet de recherche International et interdisciplinaire "European Language Diversity for All" (ELDIA)", auquel ont participé l'université Johannes Gutenberg de Mainz, l'université de Helsinki, celle de Vienne, l'institut pour la paix des îles Åland, l'université de Maribor, celle de Stockhom et celle de Tartu, a été financé par le $7^{\mathrm{e}}$ programme cadre de la Commission européenne. Loin de se concentrer uniquement sur les caractéristiques sociolinguistiques ou écolinguistiques de certaines communautés, ELDIA s'est donné comme objectif d'obtenir des données comparables sur des langues non dominantes vivant dans un espace multilinguistique, qui seraient généralisables et applicables à d'autres langues de groupes plurilinguistiques dans le cadre d'études de durabilité (ainsi que d'études prospectives). C'est pourquoi les groupes cibles d'ELDIA ont été choisis de sorte à proposer un spectre aussi large que possible de groupes linguistiques (minorités autochtones, nouveaux migrants, langues sans norme établie, etc). Les langues d'ELDIA ainsi que leur localisation figurent sur la carte suivante (Figure 1). 


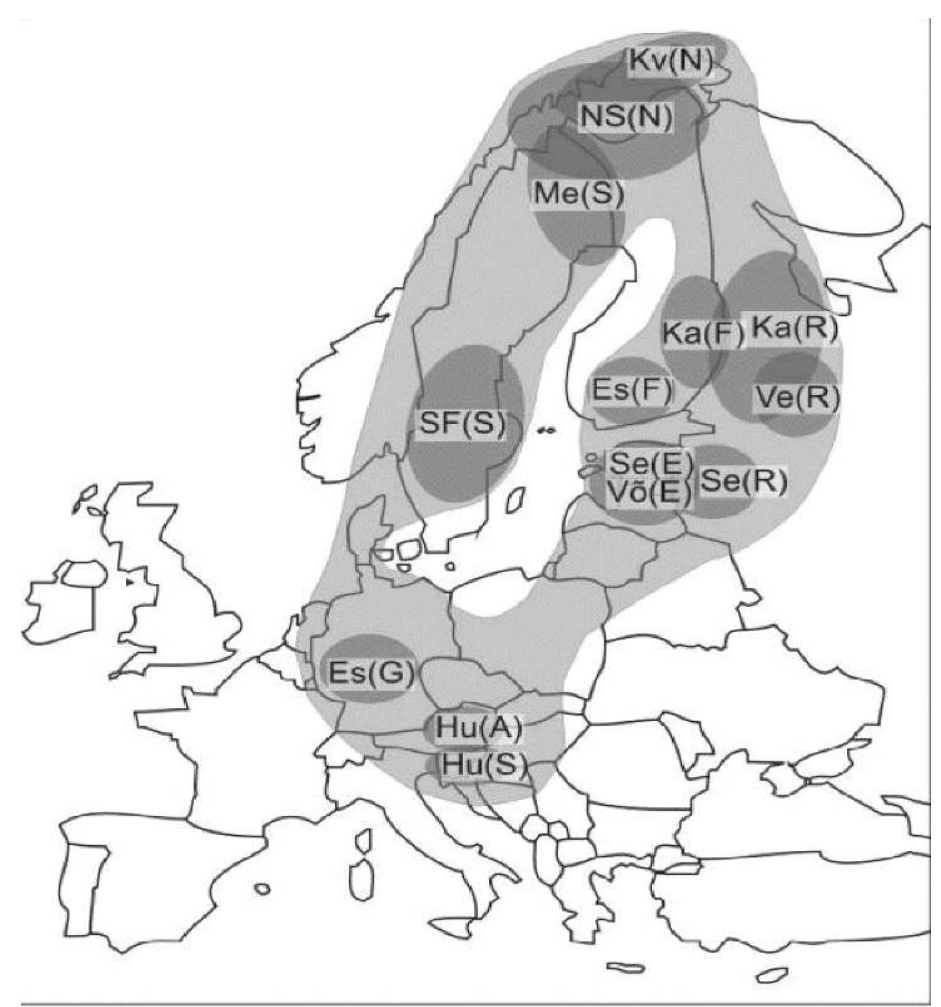

Les langues concernées par le projekt ELDIA : le kainu (ou kvène) en Norvège $K v(N)$; le same du Nord en Norvège NS(N); la meä keel de Suède $\mathrm{Me}(\mathrm{S})$; le finnois de Suède SF(S) ; le carélien en Finlande $\mathrm{Ka}(\mathrm{F})$; le carélien en Russie $\mathrm{Ka}(\mathrm{R})$; l'estonien en Finlande $\mathrm{Es}(\mathrm{F})$; le vepse en Russie Ve(R) ; le võru en Estonie Võ(E) ; le seto en Estonie Se(E) ; le seto en Russie SE(R) (laissé de côté) ; l'estonien en Allemagne $\mathrm{Es}(\mathrm{G})$; le hongrois en Autriche $\mathrm{Hu}(\mathrm{A})$; le hongrois en Slovénie Hu(S).

2 Toutes ces langues peuvent être considérées comme étant plus ou moins en danger. Le principal objectif d'ELDIA était d'élaborer un baromètre (EuLaViBar) de la vitalité des langues européennes. Les terrains ont été menés dans toutes les communautés suivant les mêmes principes et des méthodes similaires. La recherche portait sur le contexte d'utilisation de la langue à l'aide d'enquêtes, d'entretiens individuels et avec des groupes cible, d'analyses du droit et des média et aboutissait à l'élaboration d'un baromètre. Pour les entretiens et les enquêtes, nous avons déterminé un ensemble de locuteurs du seto, à savoir tous les habitants permanents du Setomaa estonien âgés de 18 ans et plus. Les terrains ont eu lieu à l'hiver et au printemps 2011. L'objectif était d'interroger trois cents personnes, et nous sommes finalement parvenus au nombre de 294. L'échantillon est représentatif du point de vue de l'âge et du sexe. L'enquête se composait de 63 questions (en seto et en estonien) réparties dans les blocs suivants : âge, lieu de naissance, formation et profession ;

- Comment et quand la personne a-telle appris le seto et l'estonien et quelles langues elle utilise/utilisait avec les membres de sa famille ;

- Maîtrise de la langue (comment la personne évalue sa maîtrise du seto, de l'estonien, de l'anglais et d'autres langues et comment elle les utilise dans différents domaines et milieux, par exemple à la maison, au travail, dans la rue, dans les commerces) ;

- Ce que la personne pense de l'utilisation du seto et de l'estonien avec divers groupes de personnes, de leur mélange voire de leur confusion dans la conversation; comment elle caractérise les différentes langues et ce qu'elle pense de leur «bon usage »; 
- Ce qu'elle pense de l'utilisation du seto dans la sphère publique ;

- Comment elle utilise les produits médiatiques et culturels (journaux, radio, télévision, internet, livres, films, etc) et comment elle produit des textes dans les différentes langues (lettres, journaux, textos, etc). d'entretiens et que je croiserai avec des informations sur le contexte de l'utilisation linguistique issues de sources secondaires. Je présenterai également les résultats du baromètre et du dernier recensement.

\section{Les Seto et le seto}

5 L'habitat traditionnel des Seto se trouve à la frontière de la République d'Estonie et de la Fédération de Russie ; conformément à la subdivision administrative actuelle, il s'agit des communes (vald, en estonien) de Mikitamäe et de Värska dans le district de Põlva, des communes de Meremäe et de Misso dans le district de Võru ainsi que du raïon de Petseri en Russie. On trouve aussi des Seto dans les grandes villes d'Estonie et ailleurs, par exemple en Sibérie orientale (voir aussi Juhkason et al. 2012). D’après le recensement de la population et des immeubles de 2011 plus de 12000 habitants permanents de l'Estonie ont reconnu parler la langue (le dialecte) seto ; la plupart d'entre eux vivent en dehors de l'espace traditionnel de la langue (Figure 2). Dans l'oblast' de Pskov, le nombre de Seto, dans les 60 dernières années, est passé de 6000 à deux ou trois centaines, surtout en raison de l'émigration (Manakov, 2004, p. 209). Celle-ci, estime-t-on, est due avant tout à la faible qualité de la vie et à l'absence de scolarisation en langue estonienne. De nos jours, les Seto s'identifient pour la plupart aussi bien comme Seto que comme Estoniens (Eichenbaum, 1998) et probablement que l'état de la communauté est aujourd'hui davantage dû à l'émigration et au vieillissement de la population qu'à la modernisation et aux changements de frontière qui la dépasse. Bien que nombre de Seto fêtent les fêtes religieuses, leur sens profond reste vague pour les jeunes (Semm, Palang, 2004). Toutefois, la religion et les symboles religieux demeurent importants pour la création de l'identité seto, même si aujourd'hui la plupart d'entre eux s'en sont éloignés.

Les dialectologues estoniens considèrent le seto comme un ou plusieurs des dialectes du võru. Même si les langues parlées seto et võru sont interintelligibles, leurs locuteurs établissent une claire démarcation entre leurs communautés linguistiques. Entre le seto et l'estonien écrit les différences se manifestent à tous les niveaux (voir par exemple Mägiste, 2007 ; Pajusalu et al., 2009). 


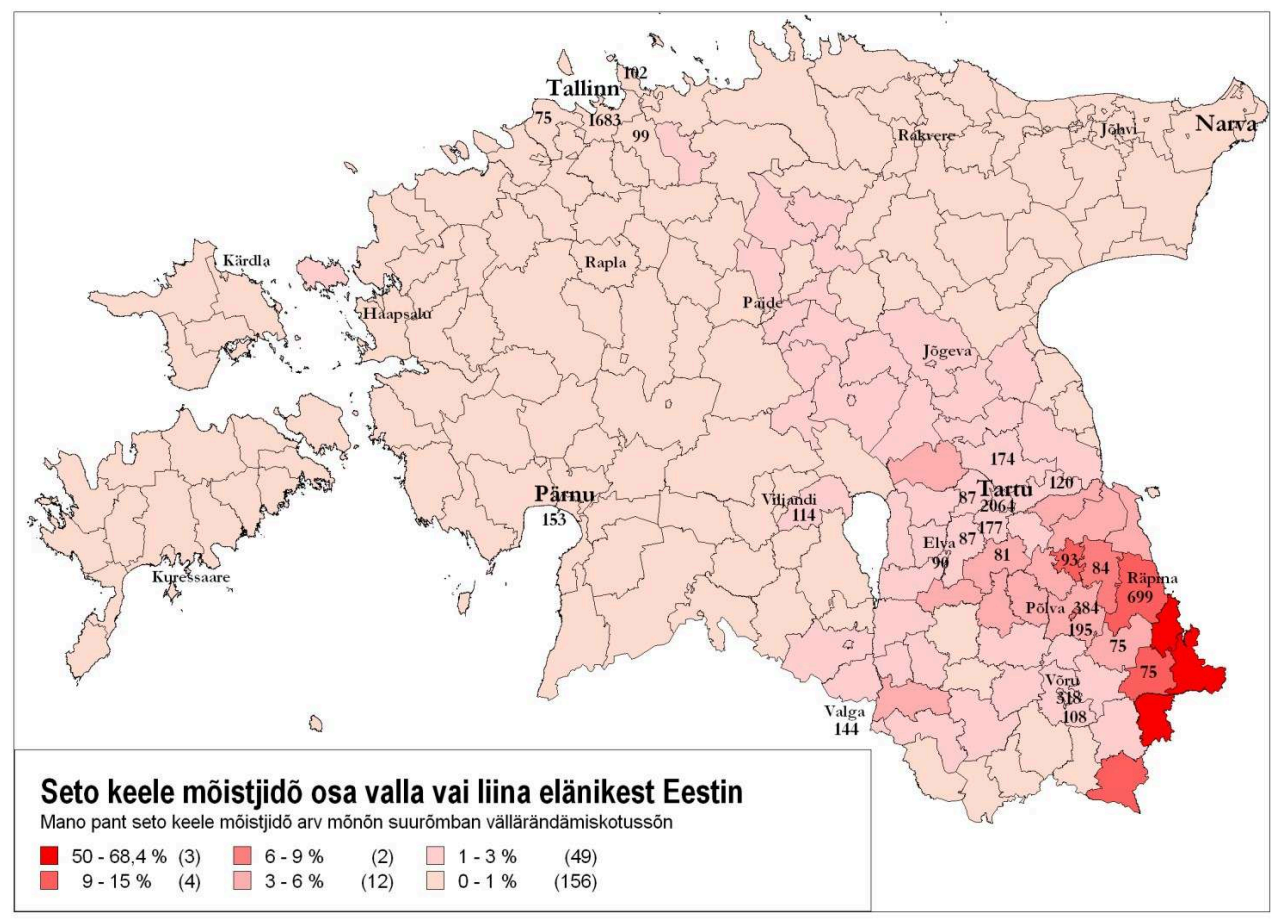

Part de la population comprenant le seto.

(Recensement de la population et des immeubles 2011)

Auteur : Evar Saar

\section{Les domaines d'utilisation du seto}

7 Le seto est utilisé dans les média imprimés et électroniques (par exemple dans des émissions radio, des films et des séries documentaires), dans des œuvres littéraires, dans des créations de toutes sortes (théâtre, musique), dans d'autres situations formelles et non formelles et naturellement il sert, comme avant, de langue domestique pour beaucoup de familles. Depuis 2007 on entend toutes les semaines des courtes informations en seto sur les ondes de la radiodiffusion estonienne. Le journal Setomaa paraît depuis 1995 et est accessible également en ligne. Si, avant 2012, Setomaa était bilingue, depuis juillet 2012 il parait uniquement en seto. Une autre édition en seto est la revue annuelle Peko Helü (2006). On publie de plus en plus de créations originales en seto. C'est en 1995 qu'est paru à Kuopio l'épopée seto Peko composée entre 1927 et 1930 par Anne Vabarna et Paulopriit Voolaine (Kuutma, 2004). C'est en 2010 qu'a été fondé l'Institut seto, qui, en 2013, a commencé à publier la collection „Seto kirävara” en seto (par exemple les évangiles de Matthieu, Marc, Luc et Jean, la deuxième édition de l'épopée Peko, des recueils de chants, etc.). À cela il faut ajouter le recueil d'histoire Setomaa 2, dont les articles ont des résumés en anglais, en russe et en seto.

En 2005 et 2006 toute l'Estonie a été marquée par la représentation de Taarka, une pièce en plein air sur le sort de la chanteuse seto Hilana Taarka (Darja Matrejeva, 1856-1933). Quelques années plus tard, en 2008, est sorti le premier film à sujet seto, lui aussi intitulé Taarka. Ces dernières années, plusieurs pièces à sujet seto et en seto ont vu le jour (par exemple, dernièrement, Non-stop seto joué par le théâtre traditionnel Taarka). 
Sur le net (par exemple sur YouTube) on trouvera des morceaux de musique traditionnelle et plus moderne, comme des concerts des chorales de leelo et des morceaux joués par le groupe ethnopop les Zeto, populaire dans toute l'Estonie, qui permettent à des jeunes aux racines seto de relier le chant et les instruments traditionnels seto aux rythmes contemporains. On trouve aussi sur les réseaux sociaux quelques groupes centrés sur les Seto et le Setomaa (par exemple Seto kiil, Setomaa OTT sur Facebook).

9 Le seto est enseigné dans une moindre mesure dans les écoles de Meremäe, Misso, Mikitamäe et Värska, et la langue est utilisée aussi dans beaucoup d'activités extrascolaires. Depuis début 2013 une école pour enfants seto a été ouverte à Tallinn, appelée Seto Latsi Kuul. L'enseignement du seto est soutenu par des publications et des enregistrements. un nouveau livre de lecture seto a vu le jour en 2009, où l'on trouve des récits, des poèmes et des pièces de théâtre aussi bien dues à des écoliers parlant le seto comme deuxième langue qu'à la plume d'auteurs expérimentés. Cette édition a été suivie, en 2012, par un abécédaire seto.

\section{La position juridique du seto}

10 La position de l'estonien en Estonie est reflétée par la Constitution de la République d'Estonie :

[La Constitution de la République d'Estonie] se doit de garantir la préservation de la nation, de la langue et de la culture estonienne à travers les temps.

11 La constitution établit également le statut de l'estonien en tant que langue officielle de l'Estonie (art. 6). En principe, la protection constitutionnelle de l'estonien s'étend aussi aux formes régionales de la langue, par exemple au seto, qui est considéré comme une partie de l'estonien, de même que l'estonien écrit normé et la langue estonienne des signes. Il convient cependant de distinguer l'usage officiel de la langue, c'est-à-dire l'usage dans l'accomplissement des tâches des pouvoirs publics, qui doit correspondre à l'estonien écrit, et les autres cas d'utilisation publique (Meiorg, 2012).

12 La loi sur la langue contient en plus deux dispositions nouvelles concernant les dialectes. Tout d'abord, l'État estonien doit assurer la protection, l'utilisation et le développement des formes régionales de l'estonien (ci-dessous, les dialectes) (art. $3 \S$ 3). Deuxièmement, dans les zones traditionnelles d'utilisation d'un dialecte, il est possible d'ajouter à un texte écrit en estonien littéraire un texte de même contenu en dialecte (art. $4 \S 1$ ). Avec l'adoption de la nouvelle loi, la cohérence de la réglementation linguistique a considérablement augmenté. Les droits liés au concept de « formes régionales de l'estonien » ont aussi été explicités. En même temps, on a vu émerger la mise en œuvre d'un concept entièrement nouveau, celui de "dialecte", dont l'assimilation aux "formes régionales" a réduit la cohérence terminologique (Meiorg, 2012).

\section{Sur les résultats de l'enquête d'ELDIA, avec des extraits d'entretiens}

13 D'après l'enquête, près de la moitié des personnes interrogées considèrent l'estonien comme leur langue maternelle, contre moins des deux tiers, qui considèrent aussi le 
seto comme langue maternelle. Parmi ces derniers, on a pris en compte aussi ceux qui ont estimé avoir deux langues maternelles, l'estonien et le seto, ainsi que les trente qui ont considéré comme leur langue maternelle le võru, le võro ou le võro-seto. Une personne a répondu que sa langue maternelle était l'estonien, mais la langue de la maison le seto. Un jeune informateur a expliqué pourquoi il considère l'estonien comme sa langue maternelle :

Tout simplement, je le parle davantage.

(Homme entre 18 et 29 ans, entretien individuel)

14 À la question de savoir s'il était difficile d'établir sa langue maternelle, un jeune informateur a répondu sans ambages :

En fait, elle ne l'est pas car moi, je suis dans l'estonien tout le temps et mes parents parlent seto et mes grand-parents, oui, aussi le seto [...], le seto est pour moi plutôt une langue de loisirs.

(Homme entre 18 et 29 ans, entretien individuel)

Bien que pour beaucoup le seto soit la langue principale de la maison, celle de leurs parents et de leurs grands-parents, seul un quart des personnes interrogées le parle avec la génération suivante. Près des deux tiers prétendent que leurs grands-parents maternels parlaient avec eux en seto quand ils étaient enfants. Un peu moins nombreux sont ceux qui disent que dans leur enfance leur mère leur parlait seto. Les deux tiers prétendent que leur père leur parlait seto quand ils étaient enfants. Une militante linguistique, à la question de savoir avec qui il parle seto, a répondu :

Comme ci comme ça [...], avec les enfants, ce sera plutôt la langue littéraire ${ }^{2}$.

(En seto, femme, entretien avec le groupe des militants)

\section{La connaissance du seto et de l'estonien}

Les résultats pour cette question sont très importants, car c'est la première fois qu'une estimation subjective de la connaissance de la langue seto a été tentée. Un peu moins des trois quarts a estimé comprendre le seto couramment, un peu moins d'un cinquième estime bien la comprendre et près de $6 \%$ et $3 \%$ ont répondu respectivement «modérément» et «mal » (Figure 3). Un peu moins des deux tiers estiment le parler couramment. Un peu moins de $40 \%$ estiment le lire couramment, un quart pense «bien " le lire. Un dixième considère la maîtrise de la lecture comme mauvaise. Près de $6 \%$ ne sont pas capables de lire en seto. Si la compréhension et la capacité à parler varient suivant les groupes d'âge, les appréciations sur la lecture et l'écriture sous tout aussi faibles dans tous les groupes (Figure 4). 
Figure 3

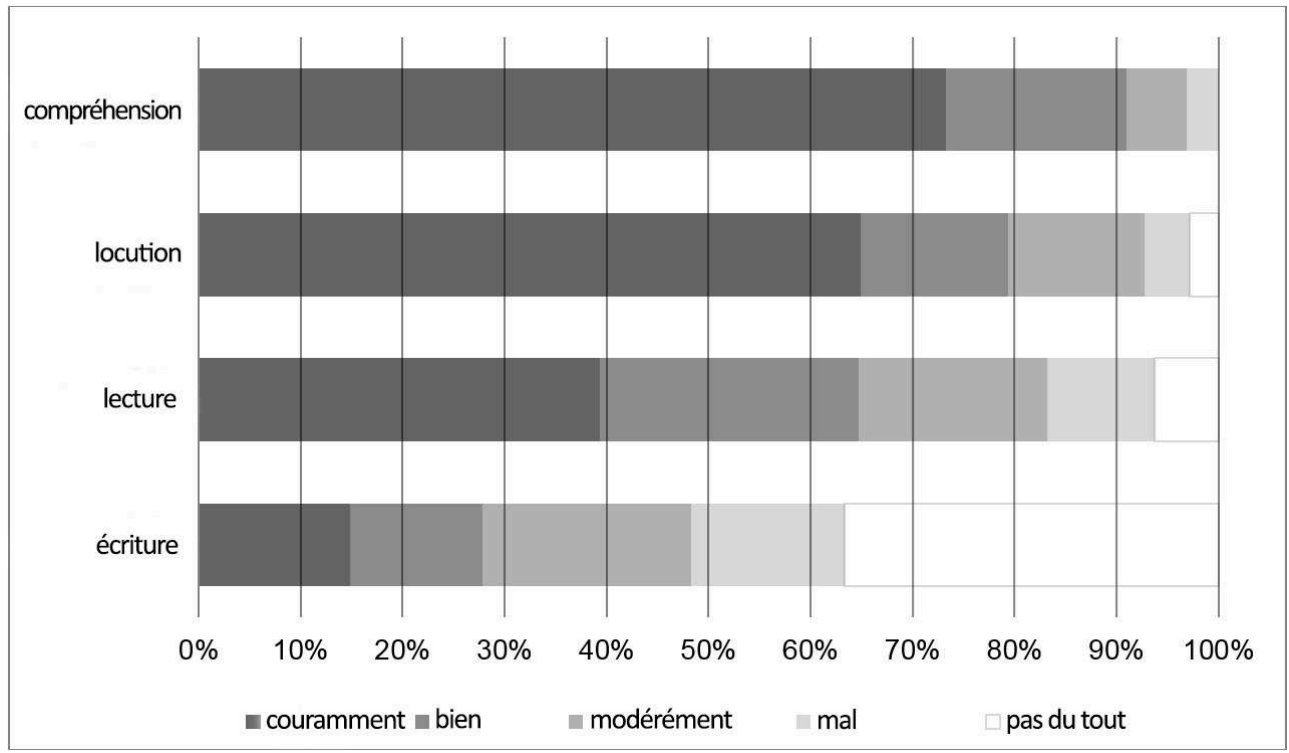

Répartition des appréciations sur la maîtrise du seto

Figure 4

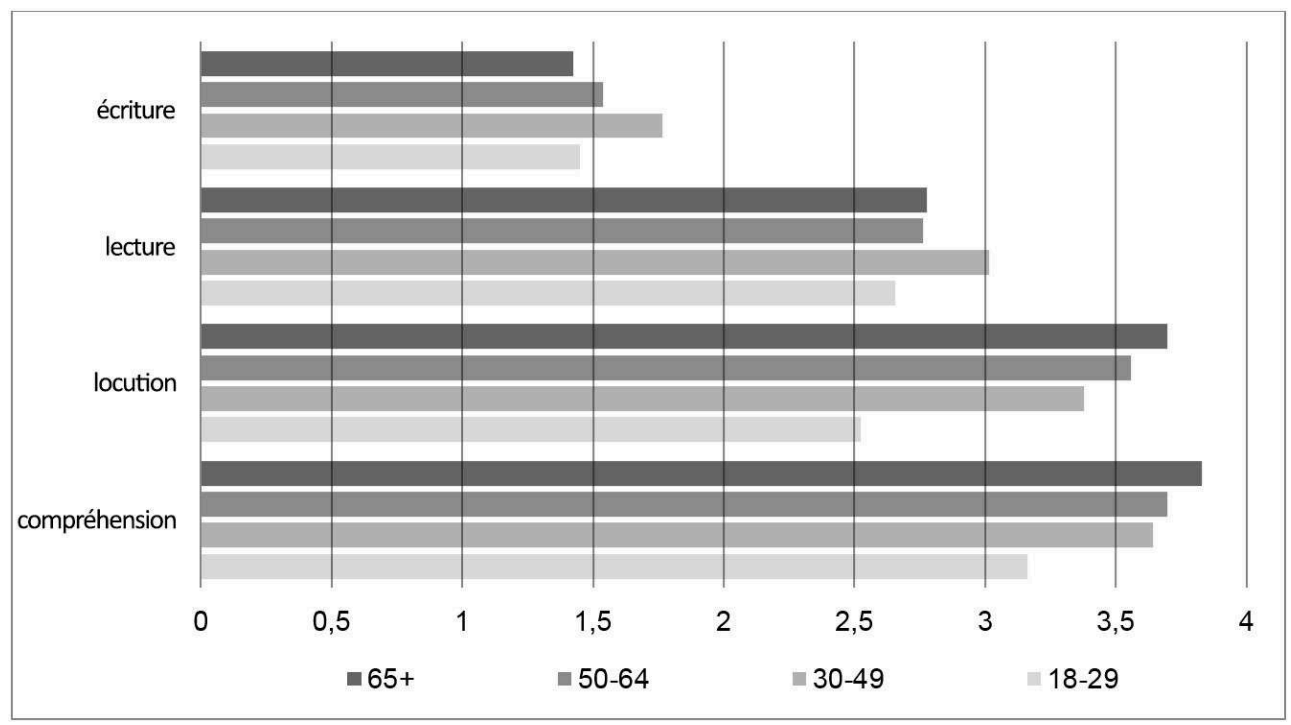

Appréciations sur la maîtrise du seto par groupe d'âge

On peut expliquer ces résultats par l'absence d'une langue normée (par exemple, d'une orthographe) enracinée et par le faible nombre des lectures possibles en seto. Les informateurs ont fait ressortir qu'il était difficile de lire en seto :

Maintenant va prendre un journal du Setomaa, essaye de lire et tu verras comme il est difficile de lire le seto.

(En seto, femme de 65+, entretien dans un groupe ciblé)

La grande majorité estime comprendre et parler l'estonien couramment. Seules quelques personnes notent mal connaitre l'estonien. Il est clair que la maîtrise courante de l'estonien est plus fréquente que la maitrise courante du seto. Mais pour 
certains, parler en estonien n'est pas inéluctable, c'est une question de choix, comme le montre un militant seto :

J'ai bien sûr des difficultés à parler estonien [...], mon estonien a été meilleur, mais maintenant, je parle toujours et par principe en seto, j'ai l'impression que je parle peu l'estonien, j'écris encore plus ou moins peut-être, mais pour parler, c'est plus difficile.

(Homme, groupe ciblé des militants)

Pour ce qui est des langues étrangères, les réponses révèlent que le russe reste la mieux connue d'entre elles; manifestement, pour les habitants de cette zone frontière, le russe est plus important que les autres langues. Plus de la moitié des personnes interrogées ont répondu " pas du tout » à la question sur leur connaissance de l'anglais, alors que plus de $7 \%$ ont noté la même chose pour le russe. Naturellement, les estimations de la capacité à parler et à écrire l'anglais et le russe se sont avérées encore inférieures.

\section{Domaines d'utilisation de la langue.}

Il semble bien que le seto soit une langue domestique, que l'on utilise avec les proches dans des situations informelles, familières (Figure 5). Environ $45 \%$ des locuteurs l'utilisent toujours à la maison, un peu plus d'un tiers toujours avec les voisins et moins d'un tiers toujours dans la communication avec la famille, même éloignée. La raison pour laquelle ce dernier chiffre est légèrement moindre tient peut-être aux migrations. Par exemple, une informatrice nous a dit :

Ma famille à moi est partie ailleurs [...] et là, à Tallinn, ils parlent la langue littéraire.

(Femme, entretien avec le groupe des militants)

21 La plupart parle seto à la maison :

Mes parents et tous, nous, à la maison, on ne parle pas estonien - comme je suis né dans la région de Petseri et là on n'utilise pratiquement pas l'estonien.

(Homme de 30-39 ans, groupe ciblé)

Figure 5

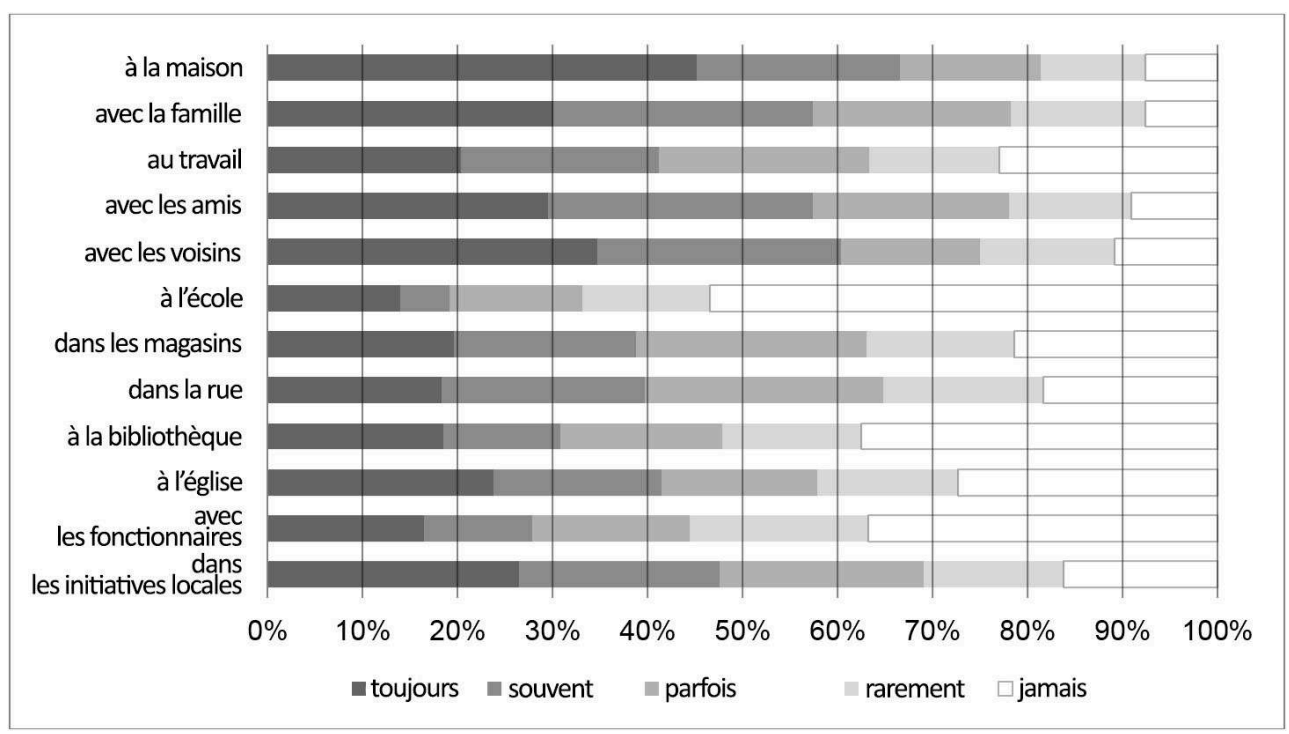

Le seto dans différents domaines d'utilisation 
Figure 6

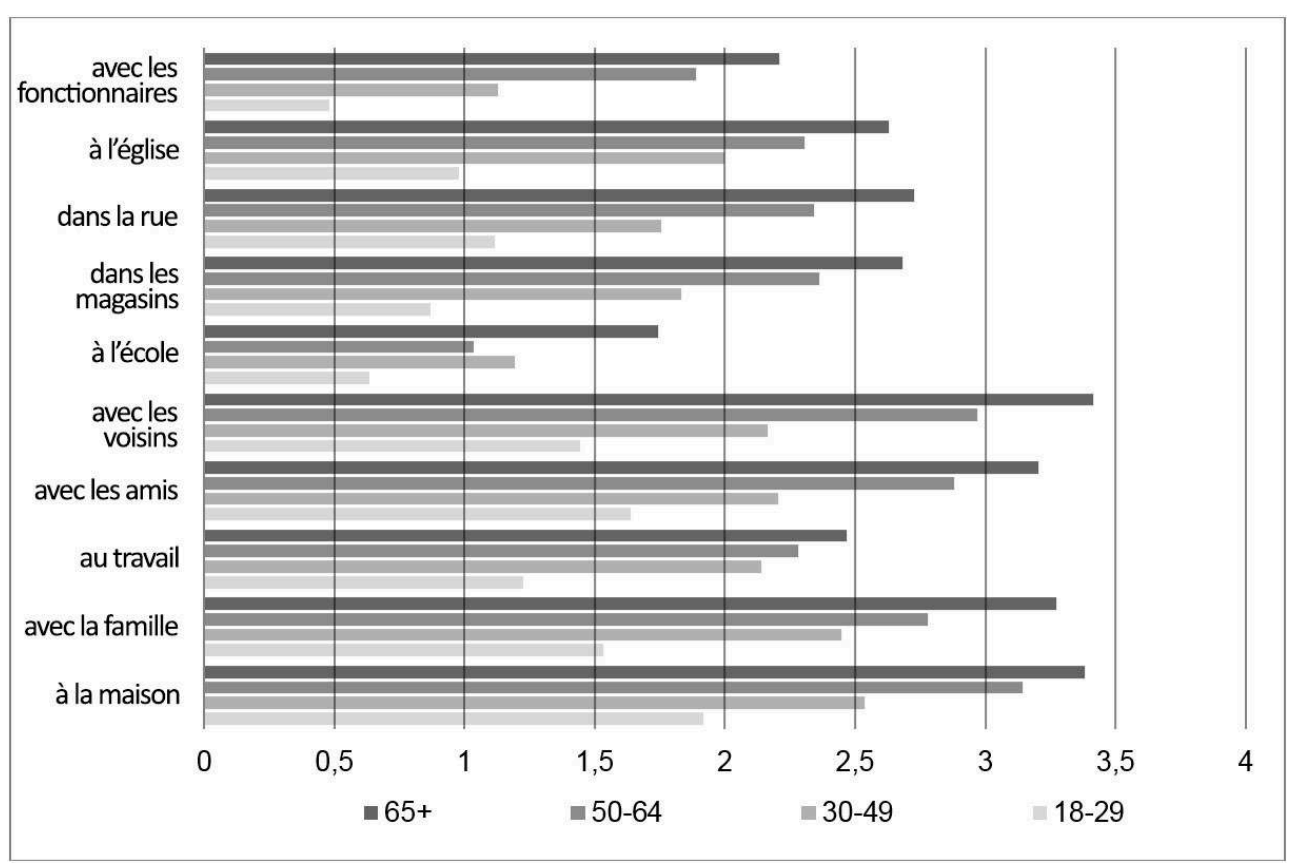

Le seto dans différents domaines d'utilisation par groupes d'âge

Le domaine d'utilisation (par exemple, le travail, la ville, en opposition à la campagne) et l'appartenance générationnelle expliquent largement l'usage de la langue (Figure 6). À la question de savoir comment elle utilise les différentes langues dans la vie quotidienne, une informatrice a donné la réponse suivante :

Oui [...], ma grand-mère est [...] de là-bas, tout de suite derrière la frontière [...]. Je chante aussi dans une chorale seto [...] et je comprends la langue et je peux l'utiliser pour parler mais comme je ne peux pas l'utiliser au travail [...] et oui [...] ailleurs aussi et alors [...] dans certains milieux peut-être [...] oui, quand nous sommes ensemble avec la famille par exemple à la campagne, n'est-ce pas, mais en général je suis avec la langue littéraire.

(Femme 30-49, groupe ciblé)

On doit constater que :

Eh bien en ville elle ne sert à rien.

Disons que oui [...] jusqu'à Tartu, une personne âgée comprendra sans problème.

(Homme, 30-49 ans, entretien individuel)

\section{Dans quels domaines faudrait-il utiliser le seto?}

Pour ce qui est du Parlement, de la télévision et d'Internet, les différences intergénérationnelles sont minimes (Figure 7). La grande majorité des personnes interrogées soit doutent de la valeur marchande du seto (sur le marché du travail: permet d'avoir un salaire majoré, facilite la carrière ou le changement de travail, accroît les chances sur le marché de l'emploi) soit n'en voient aucune. En revanche, l'estonien apparaît comme indispensable sur le marché du travail. Près de $60 \%$ des personnes interrogées ont été d'accord pour estimer que sur le marché du travail la connaissance de l'anglais est indispensable et permet de profiter des avantages 
énumérés ci-dessus. Pour le groupe de contrôle également l'anglais a une véritable valeur sur le marché.

Figure 7

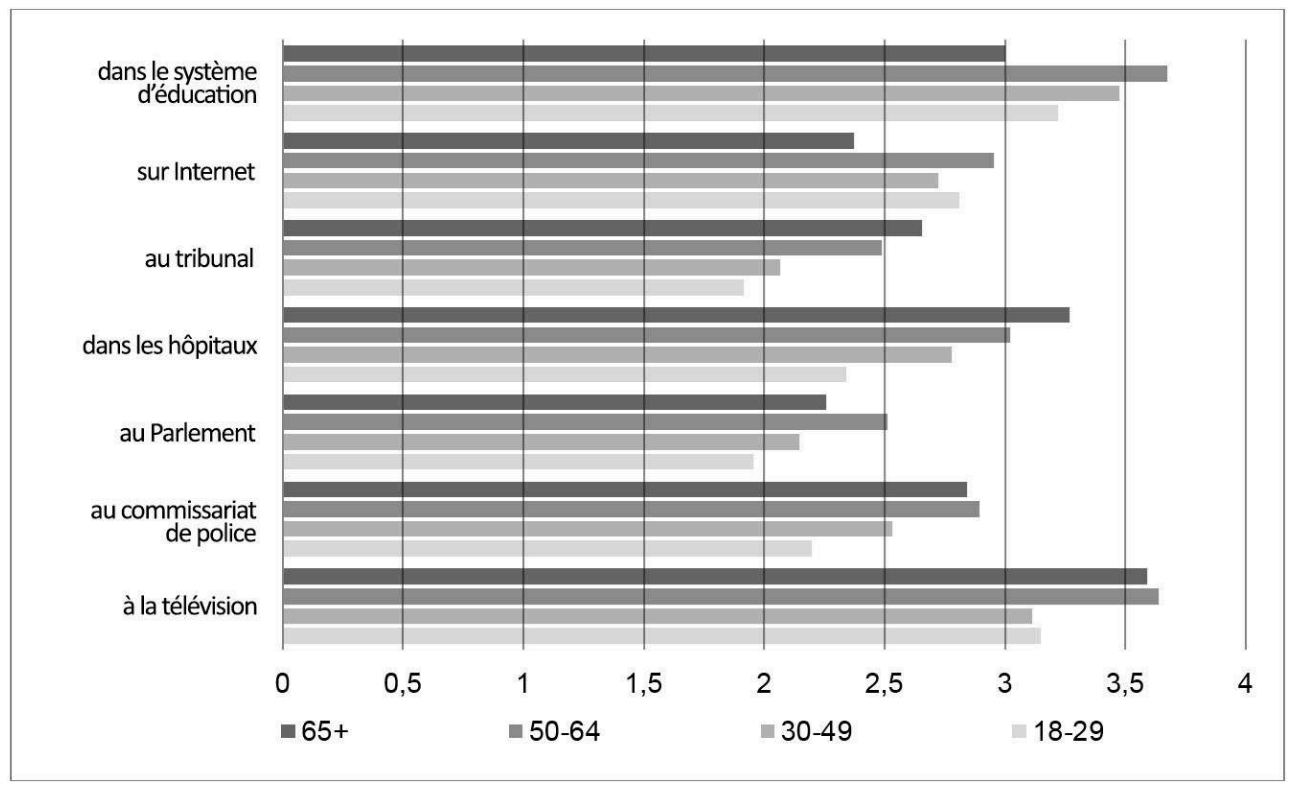

Dans quels domaines faudrait-il utiliser le seto ?

\section{Aménagement linguistique et protection de la langue.}

La moitié des personnes interrogées n'ont pas la moindre idée de l'existence d'organisations ou d'individus qui se dédient à l'entretien de la langue seto. Plus de 43 $\%$ ont donné des noms d'institutions ou de personnes, surtout de personnes : Õie Sarv, Veera Hirsnik, Laine Lõvi, Paul Hagu, Silver Hüdsi, Raul Kudre, Tiiu Kunst, Ingrit Kala, Vello Lõvi, Margus Timmo, Ilmar Vananurm, Elli Lepasild, Inara Luigas, Aare Hõrn, Vello Jüriöö, Mirjam Nutov et Kaido Kama, mais également le journal seto Setomaa, l'institut seto, l'institut Võru, les chorales leelo, les écoles, le journal Uma Leht, les musées de Saatse et de Värska, l'Union des communes du Setomaa, diverses associations, l'assemblée des anciens du Congrès seto, etc. 
Figure 8

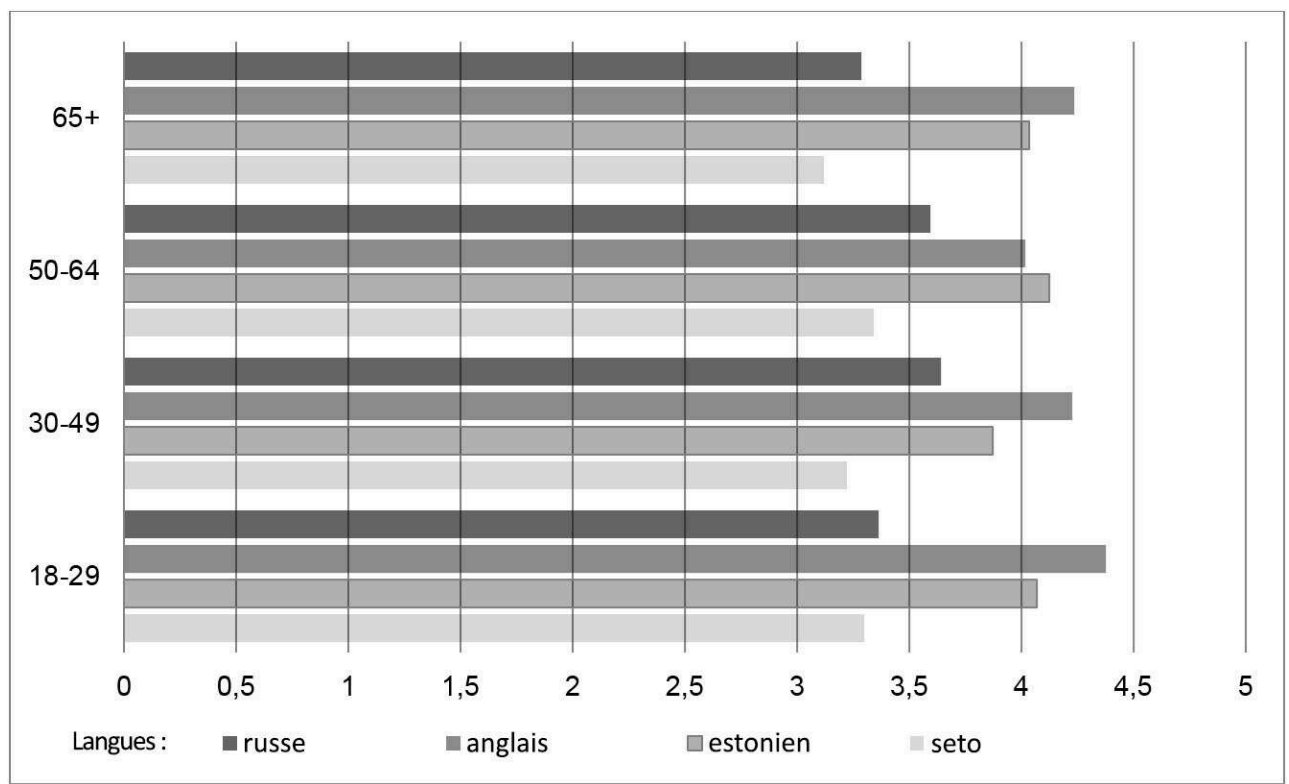

L'importance grandit dans les dix prochaines années

Figure 9

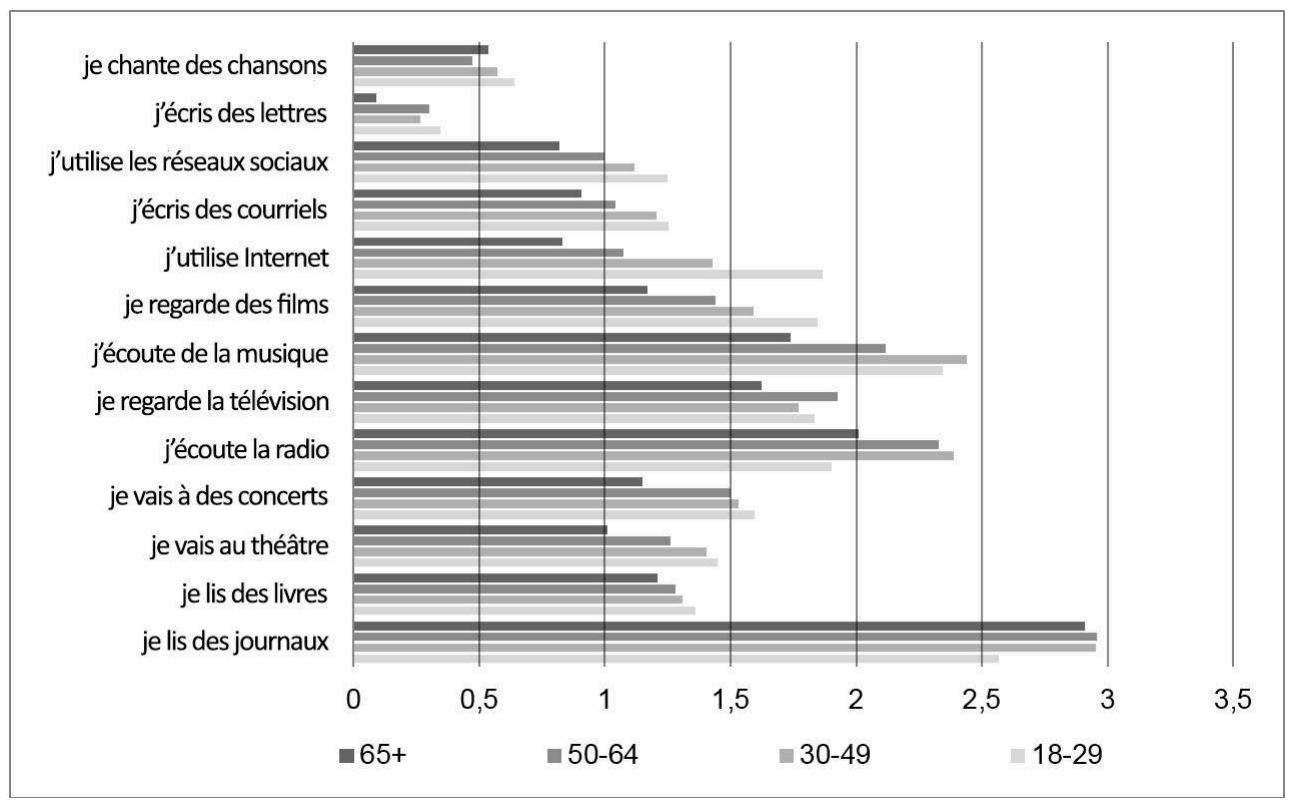

En seto, je...

De manière générale, sur l'augmentation de l'importance des langues dans les dix prochaines années, on est plutôt optimiste, sans distinctions générationnelles (Figure 8). En revanche la consommation et la production de média en seto et de culture seto (les produits dits linguistiques) se raréfient et deviennent plus modestes, surtout en raison du faible nombre de ressources en seto (Figure 9). Du point de vue de la durabilité de la langue, je veux insister sur l'importance de maintenir en Estonie du Sud-est une part plus importante de personnes comprenant le seto et aptes à donnerla 
réplique dans cette langue que celle que montre le recensement avec ses résultats (Figure 10).

Figure 10

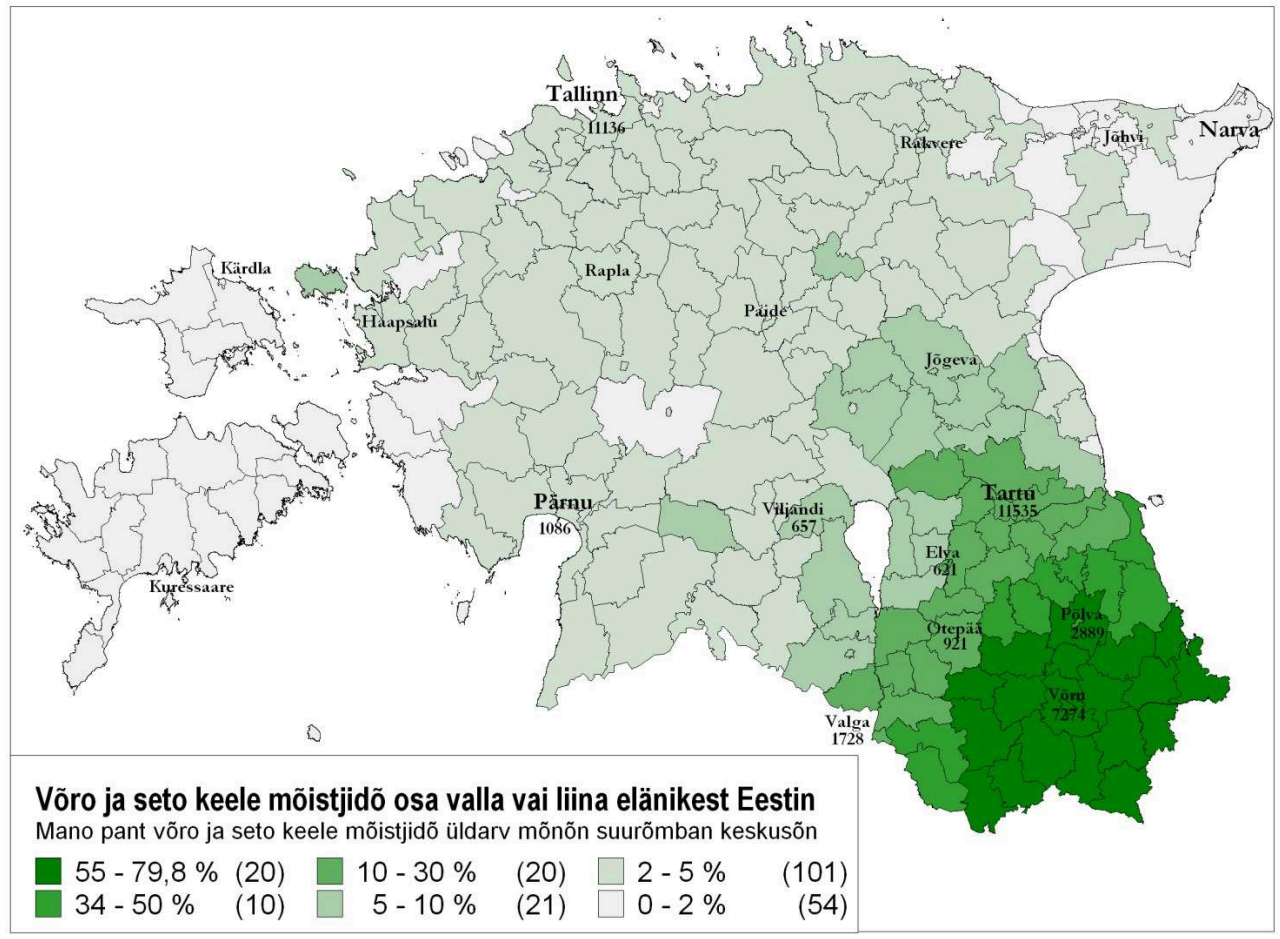

Les personnes maîtrisant le seto et le võro.

(Recensement de la population et des immeubles 2011)

Auteur : Evar Saar

\section{Baromètre de la vitalité du seto}

ELDIA part du principe que la vitalité d'une langue est mesurable et s'appuyant sur différentes études, par exemple sur les travaux de Joshua Fishman, Leena Huss, Christopher Stroud et Anna-Riitta Lindgren, mais aussi sur les rapports de l'UNESCO sur la vitalité des langues et sur le niveau du danger qui les menace $(2003,2009)$; surtout, le projet s'appuie sur une adaptation des idées de deux spécialistes d'écologie des langues, François Grin et Miquel Strubell. Les quatre zones d'intérêt du baromètre sont la durabilité, les possibilités, les souhaits et les produits linguistiques, qui à leur tour se subdivisent en quatre dimensions.

- Législation : existe-t-il ou non des textes législatifs concernant la langue, l'utilisation de la langue et la diversité linguistiques? Sont-elles soutenues, limitées ou freinées? Les locuteurs sont-ils conscients de leur législation et comment se positionnent-ils à son sujet?

- Éducation : cette dimension concerne les questions de l'apprentissage et de l'enseignement formels et informels (par exemple : niveau de formation, apprentissage de la langue, langue d'enseignement, opinions, émotions et attitudes concernant l'enseignement, etc.).

- Média : touche aux questions liées aux média - consommation de média, existence de média dans la langue minoritaire, langue des produits médiatiques et de la consommation 
médiatique, traitement des questions dominantes dans les média, média des minorités, traitement des questions des minorités dans les média dominants, etc.

- Utilisation de la langue et interaction : cette dimension touche à tous les aspects de l'utilisation de la langue dans différentes situations avec des personnes différentes.

À leur tour, ces dimensions se subdivisent en paramètres à valeurs définies. Pour leur définition, on est partis de la possibilité de mesurer toutes sortes de valeurs dans les réponses aux enquêtes sur l'échelle de la vitalité linguistique :

- 0 la langue minoritaire n'est pas utilisée, la langue n'est pas protégée, elle est sérieusement en danger.

- 1 Des signes et des critères montrent que la langue est en danger.

- 2 Des signes et des critères montrent clairement que le changement linguistique est en cours ou en progression.

- 3 Des signes et des critères montrent que la préservation de la langue a été atteinte ou maintenue ou que son niveau optimal d'utilisation et de protection est garanti.

- 4 Des signes et des critères montrent que plus que l'utilistion et le développement de la langue sont garantis à un niveau supérieur que simplement en vue d'un maintien, c'est-à-dire que le niveau de stabilité de la langue a été atteint.

Le baromètre illustre éloquemment la vitalité du seto. Sur ce baromètre, sa situation est entre "langue en danger» et "changement linguistique en cours». Certaines dimensions de l'analyse - la législation, les média et l'éducation - ont obtenu dans tous les domaines des résultats très bas, ce qui montre où sont les principaux dangers à la vitalité de la langue (Figure 11). Pour comparaisons, je présente ici aussi un exemple d'autres communautés linguistiques, concernant l'utilisation de la langue et les produits lindguistiques (tableaux 1-2). 
Figure 11

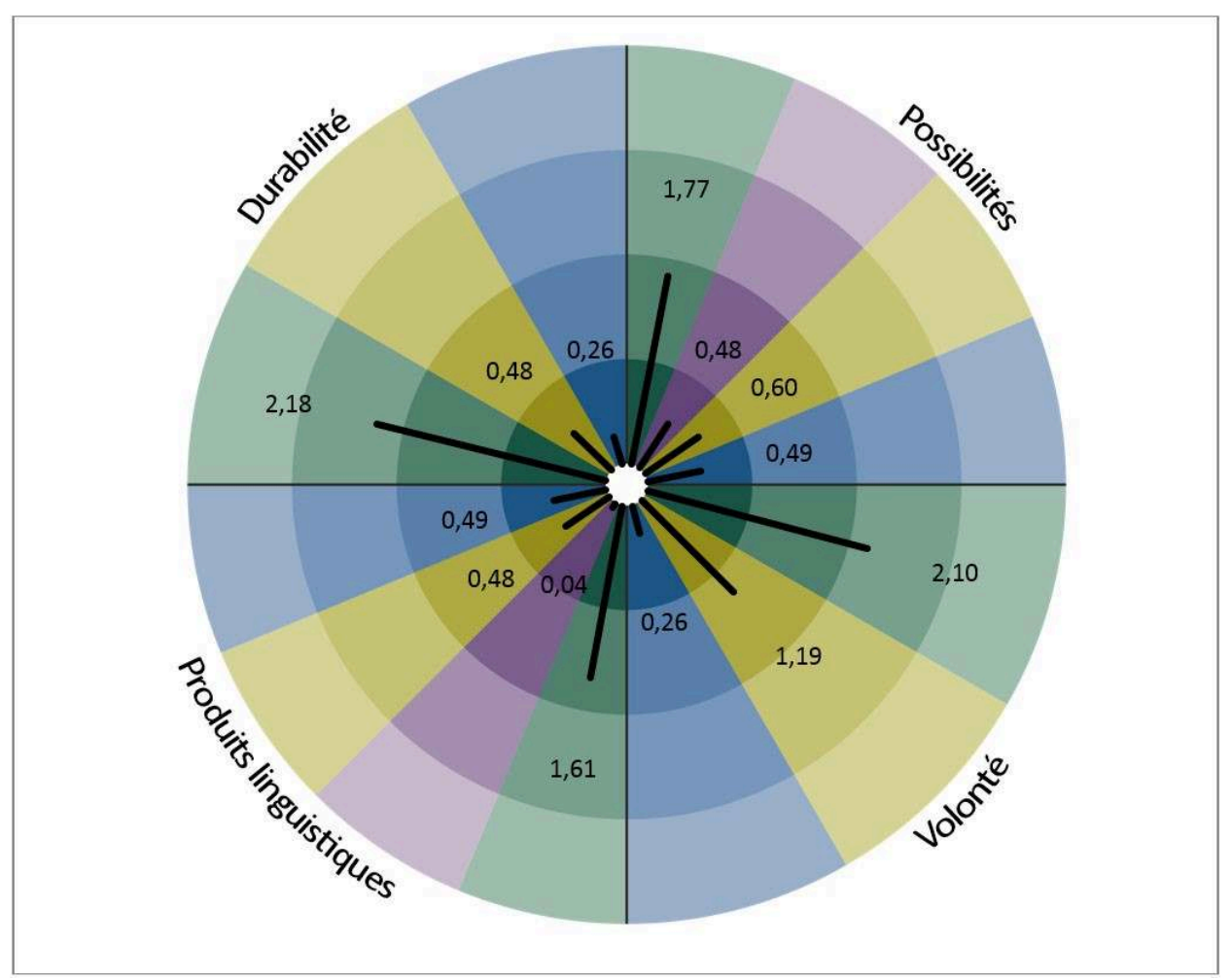

Paramètres EuLaViBari sur la vitalité du seto.

Légende : vert : utilisation de la langue et interaction ; violet : éducation ; jaune : législation, bleu : médiaEuLaViBar sur les produits linguistiques pour comparaison (Laakso et al., 2013)

\section{Tableau 1}

\begin{tabular}{|l|l|l|l|l|l|}
\hline & $\begin{array}{l}\text { Valeur } \\
\text { moyenne }\end{array}$ & $\begin{array}{l}\text { Utilisation de la langue } \\
\text { et interaction }\end{array}$ & Législation & Média & Éducation \\
\hline Estonien en Finlande & 1,717 & 1,11 & 0,5 & 1,65 & 2,66 \\
\hline Hongrois en Slovénie & 1,684 & 2,33 & 2,37 & 1,4 & 1,64 \\
\hline $\begin{array}{l}\text { Same du nord } \\
\text { en Norvège }\end{array}$ & 1,575 & 2,7 & 3,32 & 1,38 & 0,94 \\
\hline $\begin{array}{l}\text { Estonien } \\
\text { en Allemagne }\end{array}$ & 1,473 & 0,5 & 0,05 & 1,49 & 2,6 \\
\hline Hongrois en Autriche & 1,459 & 1,63 & 0,91 & 1,33 & 1,78 \\
\hline Carélien en Russie & 0,718 & 1,95 & 0,49 & 0,61 & 0,13 \\
\hline Meänkieli en Suède & 0,71 & 1,78 & 2,06 & 0,64 & 0,05 \\
\hline Vepse en Russie & 0,584 & 1,57 & 0,15 & 0,42 & 0,15 \\
\hline Seto en Estonie & 0,57 & 1,61 & 0,48 & 0,49 & 0,04 \\
\hline Võro en Estonie & 0,569 & 1,66 & 0,22 & 0,37 & 0,07 \\
\hline Carélien en Finlande & 0,515 & 1,52 & 0,14 & 0,41 & 0,04 \\
\hline
\end{tabular}




\begin{tabular}{|l|l|l|l|l|l|}
\hline Kvène en Norvège & 0,299 & 0,9 & 0,78 & 0,1 & 0,01 \\
\hline
\end{tabular}

EuLaViBar sur les produits linguistiques pour comparaison (Laakso et al., 2013)

Tableau 2

\begin{tabular}{|l|l|l|l|l|}
\hline & $\begin{array}{l}\text { Valeur } \\
\text { moyenne }\end{array}$ & $\begin{array}{l}\text { Utilisation de la langue } \\
\text { et interaction }\end{array}$ & Législation & Média \\
\hline Estonien en Allemagne & 2.679 & 2,99 & 0,05 & 1,02 \\
\hline Hongrois en Slovénie & 2.491 & 2,75 & 2,37 & 0,89 \\
\hline Estonien en Finlande & 2.465 & 2,74 & 0,5 & 0,97 \\
\hline Hongrois en Autriche & 2.432 & 2,71 & 0,91 & 0,85 \\
\hline $\begin{array}{l}\text { Same Nord } \\
\text { en Norvège }\end{array}$ & 2,02 & 2,18 & 3,32 & 0,8 \\
\hline Seto en Estonie & 1,917 & 2,18 & 0,48 & 0,26 \\
\hline Võro en Estonie & 1,628 & 1,86 & 0,22 & 0,2 \\
\hline Carélien en Russie & 1,61 & 1,82 & 0,49 & 0,36 \\
\hline Vepse en Russie & 1,597 & 1,81 & 0,15 & 0,28 \\
\hline Meänkieli en Suède & 1,556 & 1,73 & 2,06 & 0,37 \\
\hline Carélien en Finlande & 0,932 & 1,05 & 0,14 & 0,22 \\
\hline Kvène en Norvège & 0,246 & 0,27 & 0,78 & 0,05 \\
\hline
\end{tabular}

EuLaViBar comparatif : vitalité (Laakso et al., 2013)

\section{Conclusion}

Bien que le seto soit de plus en plus utilisé aussi bien dans des situations formelles que dans des cadres informels, il reste une langue de la maison, qui, tout en étant émotionnellement proche, demeure la langue des parents et des grands-parents. Près d'un quart essaye de transmettre d'une manière ou d'une autre le seto à la génération suivante, les autres laissent leurs enfants porter le fardeau linguistique. Pour beaucoup, le seto est la langue de l'environnement proche, la langue dans laquelle on parle des affaires domestiques ; l'estonien est la langue des média et de l'éducation. L'anglais est hautement estimé, mais peu connu. La plupart considère que l'anglais est essentiel pour être présent et réussir sur le marché du travail. Le partage des rôles dans la diglossie seto/estonien semble être en place depuis longtemps: la plupart des personnes interrogées ne se souviennent pas que les parents aient empêché les enfants de parler seto. La langue seto renvoie à des émotions positives. Le mélange des langues n'est pas vu comme condamnable; l'utilisation des deux langues tour à tour ou ensemble semble être la principale stratégie de transmission dans les foyers des locuteurs du seto. Une certaine incertitude législative, une représentation déficiente ou limitée dans les média dominants et la faiblesse des produits linguistiques que révèle le baromètre suggère que la vitalité et la durabilité du seto sont sérieusement en danger. 


\section{BIBLIOGRAPHIE}

EICHENBAUM Külli, 1998, Ku kavvas Setomaalõ seto rahvast jakkus. Ajaloolise Setomaa põlisasustuse säilimise võimalused [Combien de temps encore les Setos existeront au Setomaa. Les possibilités de préserver l'habitat ancestral du Setomaa historique], Võro Instituut, Võro, 202 p.

JUHKASON Grete, KALKUN Andreas, LINDSTRÖM Liina \& PLADO Helen, 2012, « Petserimaa setodest ja nende keelest 2010. 2011. aasta välitööde põhjal [Sur les Seto du Petserimaa et sur leur langue sur la base des terrains de 2010-2011] », in JÜVÄ Sullõv (ed.), Läänemeresoome piirid. [Les frontières fenniques], Võro Instituut, Võro, pp. 11-29.

KUUTMA Kristin, 2004, “Creating a Seto Epic. Oral Tradition” in Slavica, n 1, vol. 19, pp. 92-137.

MÄGISTE Julius, 2007, Seto sõnastik [Dictionnaire seto], Võro Instituut, Võro, 116 p.

MANAKOV Andrey Gennadievich МАНАКОВ АНДРЕЙ ГЕННАДЬЕВИЧ, 2004, НА СТЫКЕ ЦИВИЛИЗАЦИЙ: ЭТНОКУЛЬТУРНАЯ ГЕОГРАФИЯ ЗАПАДА РОССИИ И СТРАН БАЛТИИ [Au croisement des civilisations : Géographie ethnoculturelle de l'Ouest de la Russie et des pays baltiques], псковскиЙ ГОСДАРСТВЕННЫЙ ПЕДАГОГИЧЕСКИЙ ИНСТИТУТ Им [Institut national pédagogique de Pskov ], псков [Pskov], 294 p.

MANAKOV Andrey Gennadievich МАНАКОВ АНДРЕЙ ГЕННАДЬЕВИЧ, 2009, «ДИНАМИКА ЧИСЛЕНННОСТИ СЕТУ ПЕЧОРСКОГО РАЙОНА В ХХ - НАЧАЛЕ ХІХе ВЕКА [La dynamique des effectifs des Seto du raïon de Pečory au XXe, début XIXe siècle]», in ИЗБОРСК И ЕГО ОКРУГА. МАТЕРИАЛЫ МЕЖДУНАРОДНОЙ -НАУЧНО ПРАКТИЧЕСКОЙ КОНФЕРЕНЦИИ 20072008 ГГ. [Izborsk et son arrondissement. Matériaux d'un colloque international, 2007-2008], псковский РЕГИОНОЛОГИЧЕСКИЙ ЖУРНАЛ [Journal régional de Pskov], иЗБорск [Izborsk], pp. 40-44. MEIORG Marianne, 2012, Legal and Institutional Framework Analysis: Seto and Võro Languages, ELDIA (Working Papers in European Language Diversity), Wien, 117 p., http://phaidra.univie.ac.at/o: 127464 .

PAJUSALU Karl, HeNNOSTE Tiit, NIIT Ellen, PÄLl Peeter \& VIIKBERG Jüri, 2009, Eesti murded ja kohanimed, édition complétée, Eesti Keele Sihtasutus, Tallinn, 320 p.

SEMM Kadri \& PALANG Hannes, 2004, "Life Ways in the Setu Cultural Landscape" in Pro Ethnologia, vol. 18, pp. 49-67.

Summary of the research project ELDIA (European Language Diversity for All), 2013, Abridged version of the original English language report written by Johanna Laakso, Anneli Sarhimaa, Sia Spiliopoulou Åkermark, Reeta Toivanen, https://phaidra.univie.ac.at/view/o:304813.

\section{NOTES}

1. www.eldia-project.org

2. C'est-à-dire en estonien standard (NdT). 


\section{RÉSUMÉS}

Cet article présente les données collectées dans le cadre du projet de recherche "European Language Diversity for All" (ELDIA) à propos de l'utilisation de la langue seto. Bien qu'ELDIA ne se soit pas concentré, loin de là, sur la caractérisation de chacune des communautés linguistiques, cet article est centré sur l'utilisation dans le Setomaa du seto mais aussi des autres langues, comme l'estonien et l'anglais. Les données empiriques proviennent d'entretiens et de sondages, qui représentent les pratiques linguistiques de la population majeure dans la part du Setomaa traditionnel qui se trouve actuellement en Estonie. Les sondages ont permis de découvrir quelle langue est considérée comme première langue ou langue maternelle, quand et comment les sondés ont appris le seto, quel est, à leur avis, leur niveau de connaissance du seto, de l'anglais et des autres langues dans un barème de 1 à 5 , ce qu'ils pensent du mélange du seto et de l'estonien, comment se présente le seto authentique et qui le parle. L'enquête a par ailleurs porté sur l'utilisation des média et des produits culturels (journaux, radio, télévision, internet, livres, films) en seto, en estonien et en anglais et sur sa fréquence. On peut dire en résumé que bien que les champs d'utilisation du seto se soient élargis, le partage des fonctions entre les langues reste bien en place : d'une part la langue de l'environnement familier, utilisée quand on parle de questions domestiques, et d'autre part la langue de l'enseignement, et de l'administration; alors que l'anglais permet d'être présent sur le marché du travail et d'y faire carrière. Bien qu'il soit souvent question des Setos dans les média dominants et qu'en 2011 on ait évalué à deux ou trois mille le nombre des locuteurs de cette langue, les résultats d'ELDIA montrent que la durabilité du seto demeure fragile.

This article presents the results of a research within the ELDIA Project "European Language Diversity for All" about the use of the Seto language. While ELDIA was certainly not about the study of each community, the focus of this article is about whether and how languages, Seto, but also Estonian and English, are used in Setomaa. The empirical data come from interviews and polls representing the language practices of the permanent inhabitants, older than 18 , of the portion of the Seto core territory situated within the Estonian Republic. The questionnaire endeavoured to elicit information about which language is considered as the first tongue or mother-tongue, when and how Seto was learnt, how the respondents assess their knowledge of Seto, Estonian and English, what they think of mixing Estonian and Seto, which language and whose is the authentic Seto language. The research focused also on whether and how often people use media and cultural productions (newspapers, radio, television, internet, books, films) in Seto, Estonian and English. We may conclude that though the domains in which Seto is used are wider than they were, the distribution of functions between the languages remains the same: one is the language of the familiar environment, the language in which one speaks about domestic issues, the other is the language of education and administration, while English is important on the labour market as well as for careers. While Seto issues are quite frequent in mainstream media, and in 2011 the community of the Seto speakers is about two or three thousand persons, the ELDIA results show that the Seto language durability remains fragile.

Artiklis avatakse uurimisprojekti "European Language Diversity for All" (ELDIA) raames kogutud seto keele kasutust peegeldavaid tulemusi. Ehkki ELDIA ei piirdunud kaugeltki üksikute keelekogukondade iseloomustamisega, on selle kirjatöö fookuses, kas ja kuidas kasutatakse Setomaal seto keelt, aga ka eesti ja inglise keelt. Empiirilised andmed pärinevad intervjuudest ja arvamusküsitlusest, mis esindavad Eesti territooriumile jääva 18aastaste ja vanemate setode traditsioonilise asuala püsielanike keelepraktikaid. Küsitlusega uuriti, millist keelt peetakse 
esimeseks või emakeeleks, millal ja kuidas õpiti seto ja eesti keelt, kuidas hinnatakse 5-palli skaalal oma seto, eesti, inglise ja teiste keelte oskust ning mida arvatakse seto ja eesti keele segunemisest ning milline või kelle keel on vastajate arvates autentne seto keel. Lisaks uuriti, kas ja kui sageli kasutatakse seto-, eesti- ja ingliskeelse sisuga meedia- ja kultuuritooteid (ajalehti, raadiot, televisiooni, internetti, raamatuid, filme). Kokkuvõtteks võib väita, et ehkki seto keele kasutusvaldkonnad on küll laienenud, on keeltevaheline funktsioonide jaotus endiselt paigas: üks on koduses ümbruses ja kodustest asjadest rääkimise keel, teine haridus- ja halduskeel, inglise keelt peab enamik tööturul osalemiseks ja edenemiseks oluliseks. Ehkki setoteemalised uudised jõuavad sageli peavoolumeediasse ja 2011. aastal loendati paarteist tuhat seto keelt oskavat inimest, osutavad ELDIA tulemused, et seto keele kestlikkus on haavatav.

\section{INDEX}

Mots-clés : durabilité linguistique, langue en danger, législation, pratique linguistique, produits linguistiques, transmission, sociolinguistique, Seto, anglais, carélien, estonien, finnois, hongrois, kvène, meänkieli, russe, same du nord, seto, vepse, võru, Allemagne, Autriche, Estonie, Finlande, Kuopio, Meremäe, Mikitamäe, Misso, Norvège, Petseri, Pskov, Põlva, Russie, Saatse, Sibérie orientale, Slovénie, Setomaa, Suède, Tallinn, Värska, Võru

Keywords : endangered language, language practice, language products, language sustainability, legislation, transmission, sociolinguistics, 21th century beginning, Seto, English, Estonian, Finnish, Hungarian, Karelian, Kven, Meänkieli, Northern Sami, Russian, Vepsian, Võru, Austria, Eastern Siberia, Estonia, Finland, Germany, Kuopio, Meremäe, Mikitamäe, Misso, Norway, Petseri, Pskov, Põlva, Russia, Saatse, Slovenia, Setomaa, Sweden, Tallinn, Värska

motscleset edasiandmine, keelekasutus, keeletooted, keele suutlikkus, ohustatud keel, seadusandlus, sotsiolingvistika, 21. sajand algus, seto, eesti, inglise, karjala, kveen, meä keel, põhja saami, soome, ungari, vepsa, võru, Austria, Eesti, Idasiber, Kuopio, Meremäe, Mikitamäe, Misso, Norra, Petseri, Pihkva, Põlva, Rootsi, Saatse, Saksamaa, Sloveenia, Setomaa, Soome, Tallinn, Venemaa, Värska, Võru 